\title{
Twist and writhe of $\delta$-island active regions
}

\author{
M. C. López Fuentes ${ }^{1}$, C. H. Mandrini ${ }^{1}$ and P. Démoulin ${ }^{2}$ \\ ${ }^{1}$ Instituto de Astronomía y Física del Espacio (CONICET-UBA), \\ CC 67, Suc 28, 1428 Buenos Aires, Argentina \\ e-mail: lopezf@iafe.uba.ar
}

${ }^{2}$ Observatoire de Paris, LESIA, UMR 8109 (CNRS), F-92195, Meudon Principal Cedex, France

\begin{abstract}
We study the magnetic helicity properties of a set of peculiar active regions (ARs) including $\delta$-islands and other high-tilt bipolar configurations. These ARs are usually identified as the most active in terms of flare and CME production. Due to their observed structure, they have been associated with the emergence of magnetic flux tubes that develop a kink instability. Our main goal is to determine the chirality of the twist and writhe components of the AR magnetic helicity in order to set constrains on the possible mechanisms producing the flux tube deformations. We determine the magnetic twist comparing observations of the AR coronal structure with force-free models of the magnetic field. We infer the flux-tube writhe from the rotation of the main magnetic bipole during the observed evolution. From the relation between the obtained twist and writhe signs we conclude that the development of the kink instability cannot be the single mechanism producing deformed flux-tubes.
\end{abstract}

Keywords. Sun: magnetic fields, sun: activity, sun: photosphere, sun: corona

\section{Introduction}

Since active regions (ARs) are produced by the emergence of subphotospheric toroidal magnetic flux tubes, they generally appear on the sun as bipolar configurations in the E-W direction (Hale's law, see Schrijver \& Zwaan 2000). A small inclination (tilt angle) of the leading polarity towards the solar Equator (Joy's Law) is also observed due to the effect of the Coriolis force during the emergence (Fisher et al. 2000). There are, however, peculiar ARs with tilt angles that widely deviate from Hale's law. They are usually interpreted as the emergence of flux tubes that suffered some kind of abnormal deformation (López Fuentes et al. 2000). Among these ARs are the so-called $\delta$-spots or $\delta$-islands (an example in shown in Figure 1), which are of particular interest because they are the most active in terms of flare and CME production. Among the possible mechanisms to explain the flux tube deformations are the interaction with large scale vortices in the convection zone, the Coriolis force, and the development of a kink instability (Fan et al. 1999).

The study of the magnetic helicity properties of the flux tubes associated to peculiar regions can provide information about the mechanisms at the origin of the deformations. For instance, in the case of the kink instability there is an internal transfer of magnetic helicity from twist to writhe, so they must have the same sign. The writhe (W) of a magnetic flux tube is a measure of the deformation of its main axis as a whole, while the twist ( $\mathrm{T}$ ) corresponds to the winding of the magnetic field lines around the axis. Clearly, there are strong motivations to constrain the possible mechanisms at the origin of this kind of ARs. Although extensive statistical investigations have been done, they could not reach definitive conclusions (see e.g., López Fuentes et al. 2003, Holder et al. 2004, Tian et al. 2005). In the meantime, new mechanisms have been proposed to explain $\delta$-spots (see e.g., Archontis \& Hood 2010). We recently began to study in a "one by one" basis a 
small set of peculiar ARs to determine the signs of the twist and writhe helicities of their associated flux tubes (López Fuentes et al. 2009). Here, we extend the study to include a total of $10 \mathrm{ARs}$ with these characteristics.

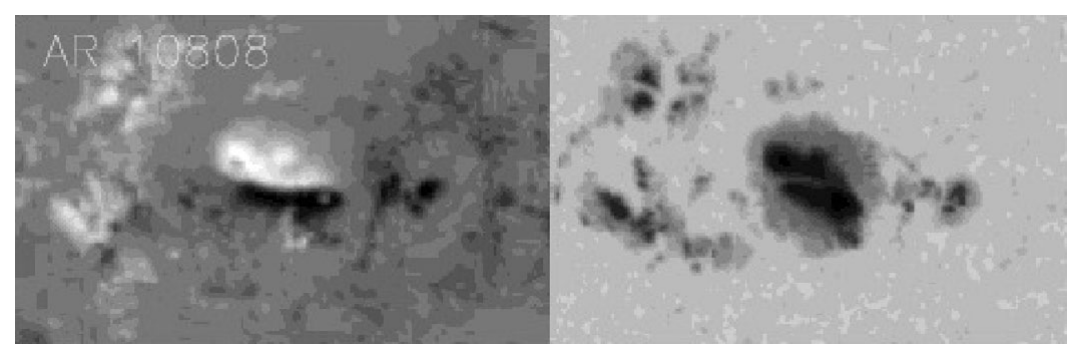

Figure 1. This is an example of $\delta$-island AR: NOAA 10808, observed during Sept. 2005. The left panel corresponds to a SOHO/MDI magnetogram obtained in Sep. 14 2005, where the high-tilt and intense magnetic strength of the main polarities can be appreciated. The right panel illustrates how in $\delta$-spots the umbrae corresponding to opposite polarities share the same penumbra.

\section{Analysis}

As we described in Section 1, we determine the signs of the twist and writhe components of the magnetic helicity for a set of peculiar ARs. In Table 1 we present a list of the studied cases, including the central meridian passage (CMP) date and the hemisphere in which the AR was observed. For the determination of the writhe we follow the evolution of the ARs main magnetic polarities using SOHO/MDI magnetograms in the way that is thoroughly described in López Fuentes \& Mandrini (2008). As the deformed magnetic flux tubes emerge through the photosphere the magnetic bipoles that form the $\delta$-spots rotate. We analyze this rotation during a total of 7 days around the CMP of the ARs. From the sense of rotation we can infer the positive or negative chirality of the flux tubes. In Figure 2 we show the rotation of the bipoles for two examples from our set. The centers of the plots correspond to the mean position of the positive polarity and the heads of the arrows indicate the relative location of the negative polarity. Initial and final dates of observation are indicated, so the sense of rotation can be appreciated and the writhe sign inferred.

For the twist sign determination we use a linear force-free field code to extrapolate SOHO/MDI magnetograms into the corona and we compare the model with coronal observations (EUV data from TRACE or soft X-ray data from Yohkoh/SXT). We use the sign of the $\alpha$ parameter from the force-free equation:

$$
\nabla \times \mathbf{B}=\alpha \mathbf{B}
$$

as a proxy for the twist sign. Using different values of the $\alpha$ parameter we obtain the mean distance between model field lines and observed coronal loops. In Figure 3 we show the mean distance to the observed loops versus $\alpha$ for the same cases shown in Figure 2 . The $\alpha$ that minimizes the distance to the observed loops provides the best sign of the AR twist.

For each of the studied ARs we compare the obtained signs of twist and writhe to determine if the development of a kink instability can be at the origin of the flux tube deformation (see Table 1). Cases with same sign of twist and writhe (60\%) are consistent with the kink instability (Table 1, last column). Cases having different twist and writhe signs cannot be explained by that mechanism. 
Table 1. Properties of the studied ARs.

\begin{tabular}{|c|c|c|c|c|c|}
\hline $\mathrm{AR}$ & Date & Hemis. & Writhe & Twist & Kink Inst. \\
\hline 8108 & 19971119 & $\mathrm{~N}$ & Neg & Pos & No \\
\hline 9026 & 20000607 & $\mathrm{~N}$ & Neg & $\mathrm{Neg}$ & Yes \\
\hline 9165 & 20000915 & $\mathrm{~N}$ & Neg & Neg & Yes \\
\hline 9415 & 20010409 & $\mathrm{~S}$ & Pos & Pos & Yes \\
\hline 9632 & 20010926 & $\mathrm{~S}$ & Neg & Pos & No \\
\hline 10314 & 20030315 & $\mathrm{~S}$ & Pos & Pos & Yes \\
\hline 10386 & 20030622 & S & Neg & Pos & No \\
\hline 10484 & 20031023 & $\mathrm{~N}$ & Pos & Neg & No \\
\hline 10696 & 20041106 & $\mathrm{~N}$ & Neg & Neg & Yes \\
\hline 10808 & 20050914 & $\mathrm{~S}$ & Pos & Pos & Yes \\
\hline
\end{tabular}

\section{Conclusions}

We study the magnetic helicity properties of a set of $10 \delta$-island or high-tilt ARs. Our main motivation is to constrain the models usually proposed to explain the deformation of emerging magnetic flux tubes. One of these mechanisms is the development of a kink instability, which implies that magnetic flux tubes must have the same sign of twist and writhe magnetic helicities. Although our set is still small for a conclusive statistical analysis, we find that only 6 of the 10 studied cases are consistent with this possibility. Therefore, other mechanisms should be considered to explain the remaining cases.

In future work we need to include more ARs in the analysis in order to obtain solid arguments for alternative mechanisms. To make a more complete inference on the magnetic structures it might be also necessary to study the photospheric evolution in more detail than just the main polarities rotation. We also plan to include vector magnetograms as an alternate source for determining the sign of the twist.
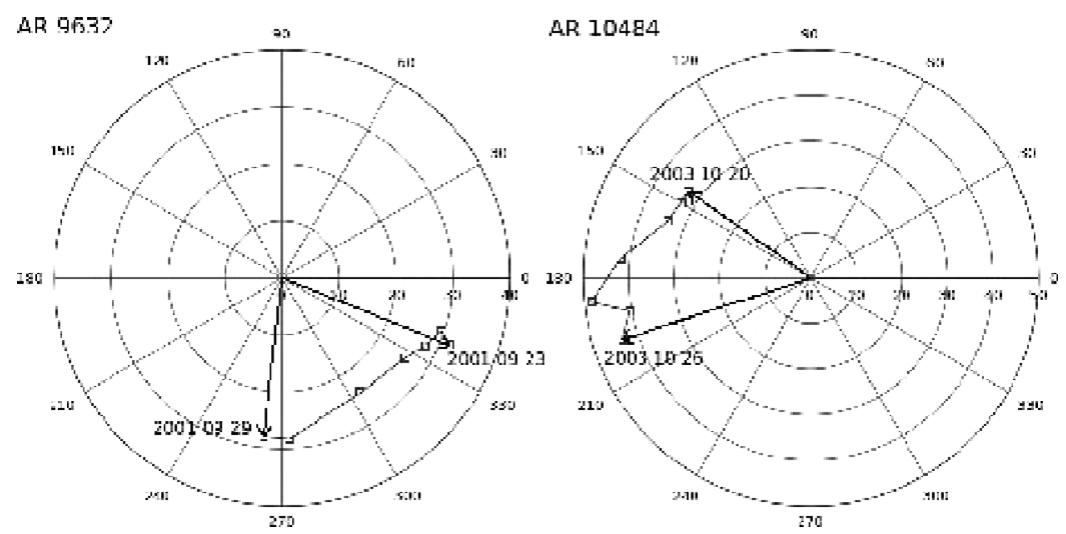

Figure 2. The polar plots show the rotation of the main bipoles during the ARs evolution for two of the studied cases. NOAA 9632 (left panel), observed in Sep. 2001, and NOAA 10484, observed during Oct. 2003. The centers of the plots correspond to the mean position of the positive polarities while the head of the arrows indicate the mean relative position of the negative polarities. From the observed senses of rotation it can be inferred that AR 9632 has negative writhe and AR 10484 has positive writhe. 

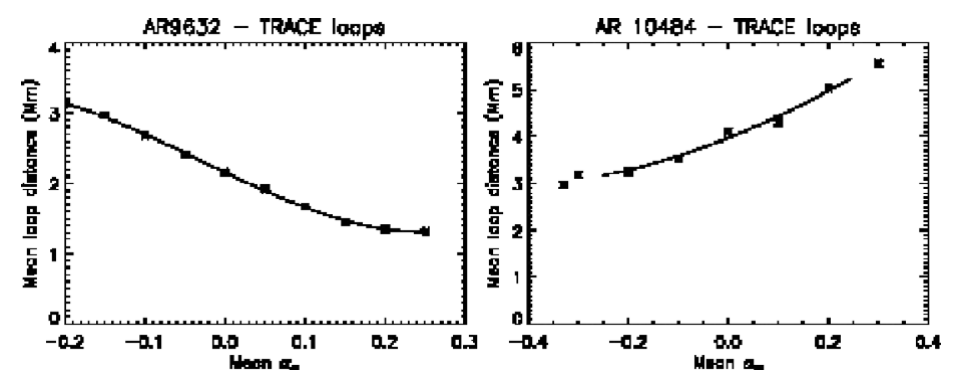

Figure 3. Mean distance from model magnetic field lines to observed coronal loops versus the force-free parameter $\alpha$. The $\alpha$ value that minimizes the mean distance provides the sign of the AR twist.

\section{References}

Archontis, V. \& Hood, A. W. 2010, Astron. Astrophys, 514, A56

Fan, Y., Zweibel, E. G., Linton, M. G., \& Fisher, G. H. 1999, Astrophys. J., 521, 460

Fisher, G. H., Fan, Y., Longcope, D. W., Linton, M. G., \& Pevtsov, A. A. 2000, Solar Phys., 192,119

Holder, Z. A., Canfield, R. C., McMullen, R. A., Nandy, D., Howard, R. F., \& Pevtsov, A. A. 2004, Astrophys. J., 611, 1149

López Fuentes, M. C., Demoulin, P., Mandrini, C. H., \& van Driel-Gesztelyi, L. 2000, Astrophys. $J ., 544,540$

López Fuentes, M. C., Démoulin, P., Mandrini, C. H., Pevtsov, A. A., \& van Driel-Gesztelyi, L. 2003, Astron. Astrophys, 397, 305

López Fuentes, M. C. \& Mandrini, C. H. 2008, Boletín de la Asociación Argentina de Astronomía La Plata Argentina, 51, 31

López Fuentes, M. C., Mandrini, C. H., \& Démoulin, P. 2009, Proceedings IAU Symposium No. 264, Solar and Stellar variability - Impact on Earth and Planets, A. G. Kosovichev Editor, 102

Schrijver, C. J. \& Zwaan, C. 2000, Solar and stellar magnetic activity / Carolus J. Schrijver, Cornelius Zwaan. New York : Cambridge University Press, 2000. (Cambridge astrophysics series ; 34)

Tian, L., Alexander, D., Liu, Y., \& Yang, J. 2005, Solar Phys., 229, 63

\section{Discussion}

Kosovichev: I have a question and comment. Question, is it seems from your model it follows that delta sunspot rotates more commonly than normal sunspots, and I wondered if this was the case. The comment is now that we have vector magnetograms from every sunspot from SDO.

FUENTES: We didn't compare the rotation of the studied delta-spots with regular sunspots. I can only say that in my experience rotation of delta-spot is fairly common. Thank you.

KLIMCHUK: So Marcelo, the force-free parameter alpha is affected by the writhe as well as the twist. So can you be confident that you're measuring the twist if you're measuring the alpha?

Fuentes: Well, I think that in fact the alpha is more affected by the twist of the flux tubes because these are two different spacial scales. This is my interpretation. You could be right in that part of the writhe could affect the coronal structure; but I think that when you consider the whole structure of the flux tube (the long term rotation of the magnetic polarities), that is the writhe. I think that the observed coronal structure inherits the sign of the twist. 